\title{
COVID-19 Repeated Convalescent Plasma Collection: Analysis of 149 Donations from 88 French Military Health Workers
}

\author{
Pierre-Louis Conan $^{a}$ Cécile Ficko $^{a}$ Marine Chueca ${ }^{b}$ Carole Rolland ${ }^{c}$ \\ Olivier Javaudin $^{b}$ Christine Bigaillon ${ }^{d}$ Guillaume-André Durand ${ }^{\mathrm{e}}$ \\ Isabelle Leparc-Goffart ${ }^{\mathrm{e}}$ Catherine Verret ${ }^{f}$ Marc Aletti $^{\mathrm{g}}$ Fabien Dutasta $^{\mathrm{h}}$ \\ Hélène Savini ${ }^{i}$ Jean-Luc Bosson ${ }^{c}$ Christophe Martinaud ${ }^{b}$ \\ aService de maladies infectieuses et tropicales - Hôpital d'Instruction des Armées Bégin, Saint-Mandé, France; \\ ${ }^{b}$ Centre de transfusion des Armées - Hôpital d'Instruction des Armées Percy, Clamart, France; 'Laboratoire TIMC- \\ IMAG, UMR, CNRS 5525, Université Grenoble Alpes, Grenoble, France; 'Service de Biologie - Hôpital d'Instruction \\ des Armées Bégin, Saint-Mandé, France; ${ }^{e}$ French Armed Forces Biomedical Research Institute, National Reference \\ Laboratory for Arboviruses, Marseille, France; fDirection de la Formation de la Recherche et de I'Innovation, Direction \\ Centrale du Service de Santé des Armées, Paris, France; ${ }^{9}$ Service de médecine interne - Hôpital d'Instruction

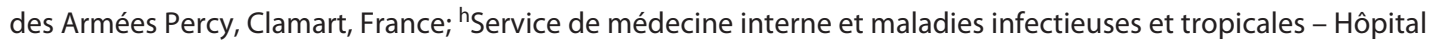 \\ d'Instruction des Armées Saint-Anne, Toulon, France; 'Service de maladies infectieuses et tropicales - Hôpital \\ d'Instruction des Armées Laveran, Marseille, France
}

\section{Keywords}

Convalescent plasma · Passive immunization · COVID-19

\section{Abstract}

Background: Passive therapy with convalescent plasma (CP) could be an effective and safe treatment option in COVID-19 patients. Neutralizing antibodies present in CP generated in response to SARS-CoV-2 infection and directed against the receptor-binding domain of the spike protein are considered to play a major role in the viral clearance. CP infusion may also contribute to the modulation of the immune response through its immunomodulatory effect. We describe for the first time the effectiveness of a CP collection protocol from repeated donations in young patients. Materials and Methods: We enrolled health service workers who experienced mild to moderate COVID-19 and from whom several donations have been collected. No minimal severity threshold and no biological cure criteria were required. Donors could return to a second plasma donation 14 days after the first donation. A minimal neutralizing antibody titer of 1:40 was considered for clinical use. Results: Eighty-eight donors were included (median age 35 [28-48] years, 41 women),
\end{abstract}

and 149 plasma products were collected. COVID-19 were mainly WHO stage 2 infections (96\%). Among the 88 first donations, $76 \%$ had neutralizing antibody titers higher than or equal to 1:40. Eighty-eight percent of donors who came for a second donation had a neutralizing antibody titer of 1:40. Median durations were 15 (15-19) and 38 (33-46) days from the first to the second donation and from recovery to the second donation, respectively. Sixty-nine percent of donors who came for a third donation had a neutralizing antibody titer of 1:40. Median durations were 16 (13-37) and 54 (4961 ) days from the second to the third donation and from recovery to the third donation, respectively. No significant difference was observed between the lgG ratio and the age of the donors or the time between recovery and donation. The average IgG ratio did not significantly vary between donations. When focused on repeated blood donors, no significant differences were observed either. Conclusion: The recruitment of young patients with a mild to moderate COVID-19 course is an efficient possibility to collect CP with a satisfactory level of neutralizing antibodies. Repeated donations are a well-tolerated and effective way of CP collection.

\section{karger@karger.com} www.karger.com/tmh

\section{Karger $\%$}

BOPEN ACCESS

\section{(C) 2021 The Author(s)}

Published by S. Karger AG, Basel

This is an Open Access article licensed under the Creative Common Attribution-NonCommercial-4.0 International License (CC BY-NC) (http://www.karger.com/Services/OpenAccessLicense), applicable to the online version of the article only. Usage and distribution for commercial purposes requires written permission.
Correspondence to:

Pierre-Louis Conan, pierre.conan@yahoo.fr 


\section{Introduction}

COVID-19 is characterized by the risk of acute respiratory failure during the second week of illness $[1,2]$. The currently available therapeutic resources are under evaluation, and only heparin and dexamethasone showed improvement with severe COVID-19 cases requiring supplemental oxygenation $[3,4]$.

Convalescent plasma (CP) is a passive immunotherapy that has often been used in respiratory virus outbreak situations: 1919 influenza pandemic, SARS-CoV-1, or MERS-CoV [5-8]. Some uncontrolled case series in the literature are in favor of a beneficial effect without serious adverse effects reported $[9,10]$. Even if no controlled trials have been published to date, there is a growing amount of data suggesting that $\mathrm{CP}$ could be an effective and safe treatment option in COVID-19 patients [11-13]. The major challenge in this therapy is obtaining donors with similar blood antigens with a high antibody titer of SARSCoV-2 [14]. However, recruitment protocol, donor characteristics, timing of collection, and the threshold for neutralizing antibodies are still under investigation [15].

PlasCoSSA is a French multicenter interventional study which aims to evaluate the effect of $\mathrm{CP}$ on survival without intensification of respiratory assistance in COVID-19 inpatients (ClinicalTrials.gov ID: NCT04372979). The donors are healthcare workers (from the French Military Health Service) who have recovered from COVID-19.

Here, we describe the CP collection protocol and the characteristics of these $\mathrm{CP}$ donors. We provide important data which could help blood donation centers to implement a CP program especially regarding recruitment efficiency and plasma quality.

\section{Material and Methods}

\section{Ethics}

This study was conducted from March to June 2020, by the French Military Blood Institute (Centre de Transfusion Sanguine des Armées, CTSA) which is the armed forces transfusion operator responsible for the transfusion support of the French Armed Forces including collection, process, qualification, delivery and hemovigilance of blood products. Blood collections take place in two collection sites (Clamart and Toulon) as well in mobile collection sites by mobile teams in military settings around the country. PlasCoSSA study (ID RCB: 2020-01,166-33) was approved by the French Ethical Research Committee (Comité de Protection des Personnes Ile-de France VIII, reference number 2004 18). The research was conducted in accordance with the Declaration of Helsinki and national and institutional standards for donor selection, plasma collection, and testing. All donors provided written informed consent.

\section{Donor Recruitment}

Recruitment

A call for recruitment was made through social networks (Twitter account of the French Military Health Service) and by internal messaging. Information for PlasCoSSA recruitment was given in all military teaching hospital and military medical centers in mainland France. Of note, regulation about personal data would not have allowed our services to contact hospital who treated these patients, or the labs which performed the tests, to gather information and directly recruit the potential donors.

Potential donors were included if they met the following criteria: (1) laboratory-proven (RT-PCR) SARS-CoV-2 infection OR medical interview consistent with a COVID-19 infection; (2) at least from day 14 after recovery (defined by the absence of symptoms (fever, dyspnea, aches, and pains) for at least 2 days).

Exclusion criteria were: (1) donors who did not meet the selection criteria for blood donation, (2) donors with a contraindication to plasma donation (age $>65$ years, past history of intolerance to apheresis collection, female without HLA antibodies); (3) donors who have not given their written consent.

No minimum severity threshold and no minimum symptom duration was required to be included. No biological cure criteria were required. Donors who met the criteria for $\mathrm{CP}$ collection were allowed to return to a second plasma donation 14 days after the first donation, according to the French guidelines.

\section{Plasma Collection}

Donor recruitment, screening, and blood specimen collection were conducted by the CTSA.

Plasma collection was performed according to routine procedure and requirements for plasma donation. Plasma collection was performed via plasmapheresis (Haemonetics $\mathrm{MCS}^{\circledR}+$, or Fresenius-Kabi Aurora ${ }^{\circledR}$ collection system). Up to $750 \mathrm{~mL}$ of plasma was collected depending on the donor's weight. The volume of replacement fluid was half of the plasma collected. All plasma products were frozen within $8 \mathrm{~h}$.

\section{Laboratory Testing}

All blood products were tested for HCV, HBV, HIV by ELISA and NAT, for HEV by NAT and for syphilis by hemagglutination according to the French national guidelines. First-time donor was tested for HTLVI/II antibodies. Donors with past history of exposure to Chagas disease or malaria were tested for antibodies against these parasites. Female donors with medical history of pregnancy were tested for anti-HLA antibodies.

All collected donor plasma samples were tested for SARSCoV-2 RNA by individual donor testing, using the Procleix SARSCoV-2 Assay (Grifols, Barcelona, Spain). The test kits are CE-IVD marked and were performed according to the manufacturer's instructions on a Panther system (Grifols, Barcelona, Spain).

$\mathrm{N}$-specific IgA and IgG antibodies were tested using the SARSCoV-2 IgG and IgA assay (Euroimmun AG, Lübeck, Germany) with a positive threshold $>1.1$ ), which use the spike protein as antigen. Tests were performed according to the manufacturers' instructions.

Neutralizing antibodies were detected using a micro-neutralization assay, adapted from neutralization testing of human antibodies to dengue viruses according to the WHO guidelines for human neutralizing antibodies against Dengue virus and whose performances were previously described $[16,17]$. Briefly, sera were 2 -fold serially diluted from 1:10 to 1:80 on 96-well plate. One hundred $\mu \mathrm{L}$ of diluted sera were added to $100 \mu \mathrm{L}$ of virus suspension containing 100 TCID50 (human SARS-CoV-2 strain BavPat1/2020, European Virus Archive global), incubated during $1 \mathrm{~h}$ at $37^{\circ} \mathrm{C}$ with $5 \% \mathrm{CO}_{2}$ and then added on Vero cells (ATCC CCL-81, $1.3 \times 105$ cells/well). After 4 days of incubation, the cytopathogenic effects were observed under a light microscope. The serum neutralizing titer was calculated as the inverse of the highest dilution resulting in an infectious reduction of $50 \%$. All experiments were performed in a BSL-3 facility. Donations with a minimal neutralizing antibody titer of 1:40 were considered for clinical use. 
Fig. 1. The proportion of neutralizing antibodies in the first, second, and third donations.

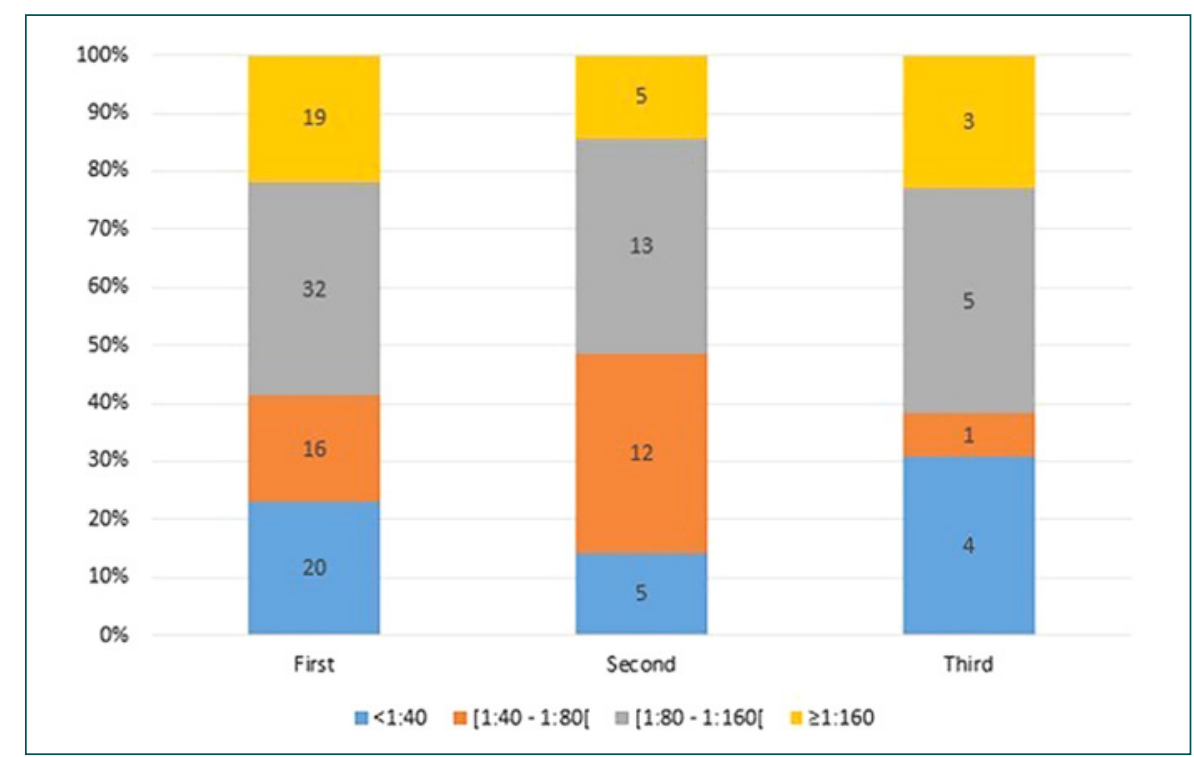

Results are evaluated semi-quantitatively by calculation of a ratio of the extinction of patient sample over the extinction of the calibrator.

\section{Clinical Data and Definitions}

We recorded demographic data (age, sex), data on COVID-19 course (duration of symptoms, OMS stage) and biological followup data (IgG, IgA, neutralizing antibody titers). COVID-19 severity was defined according to WHO progression scale.

\section{Statistical analysis}

A descriptive analysis was performed after all data had been collected and verified. Categorical variables are given as numbers and proportions. Denominators were the number of data available for each variable. Continuous variables are summarised as medians with interquartile range. Data were compiled and analysed using an EXCEL ${ }^{\circledR}$ spreadsheet (Microsoft, WA, USA), XLSTAT ${ }^{\circledR}$ software version 19.4, and GraphPad Prism version 8.0.2 (GraphPad Software, San Diego, CA, USA).

\section{Results}

Eighty-eight donors met the inclusion criteria (i.e., French Army healthcare workers who experienced a SARS-CoV-2 infection with recovery for at least 14 days).

\section{Baseline Characteristics}

The median age of the 88 donors was 35 (28-48) years, and 41 were women (female-to-male ratio 1/1.15). None had significant medical history.

All of them had a laboratory-proven COVID-19 by nasopharyngeal RT-PCR. Seventy-six (96\%) out of 79 donors with available data had WHO stage 2 infections. Only two (3\%) were treated as in-patients for a WHO stage 4 disease. One (1\%) donor had WHO stage 0 . This diagnosis was made in a tracing contact. Median duration of symptoms was 11 (7-15) days.

\section{Convalescent Plasma}

Forty-one (47\%) donors were first-time blood donors. From the 88 donors, 149 plasma products were collected. The anti-HIV 1/2, anti-HCV, syphilis testing, and HbsAg were all negative. The median number of donations was $1.7(1-3)$. The median time from the recovery to the first donation was 22 (18-30) days.

SARS-CoV-2 PCR was performed in 65 first donations and was positive in 63 (97\%) cases. Among the total 88 First donations, 73 (83\%) and 73 (83\%) were IgG and IgA positive. Seventy (80\%) were both IgG and IgA positive. Titers collected from 14 to 71 days after the onset of symptoms were tested by the neutralization assay. Sixtyseven $(76 \%)$ had neutralizing antibody titer higher than or equal to 1:40. Among them, 51 and 19 had neutralizing antibody titers of $1: 80$ and $1: 160$, respectively.

Forty-two (44\%) donors came for a second donation. Median durations were 15 (15-19) and 38 (3-46) days from the first to the second donation and from recovery to the second donation, respectively. Thirty-five out of 38 (92\%) and 32/38 (84\%) were IgG and IgA positive. Thirty out of $35(86 \%)$ had a neutralizing antibody titer of 1:40. Eighteen and 5 had neutralizing antibody titers of 1:80 and $1: 160$, respectively.

Nineteen $(22 \%)$ donors came for a third donation. Median durations were 16 (13-37) and 54 (49-61) days from the second to the third donation and from recovery to the third donation, respectively. Among them, 17/17 (100\%) and $13 / 17$ had, respectively, positive IgG and IgA. Nine out of $13(69 \%)$ had a neutralizing antibody titer of 1:40. Among them, 8 and 3 had neutralizing titers of 1:80 and 1:160, respectively. Figure 1 summarizes the proportions of neutralizing antibody titers in the first, the second, and the third donation. To test if age of donors may be related to IgG ratio, we tested IgG mean in 4 age ranges (20-29, 
Fig. 2. IgG (ratio) according to age ranges (years) of blood donors $(n=29,26,13$, and 20 , respectively).
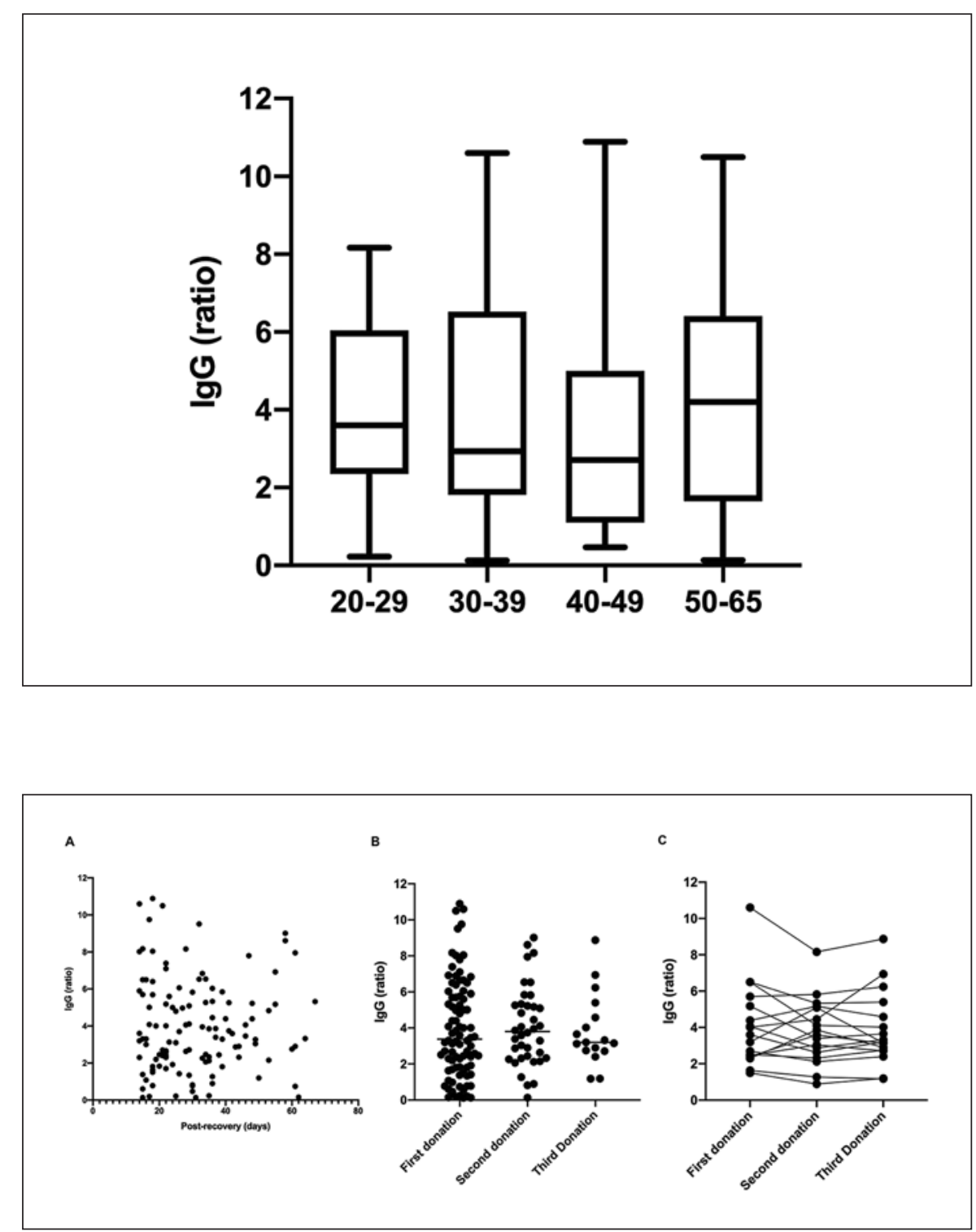

Fig. 3. Evolution of the IgG ratio according to time post-recovery and blood donation. A Correlation between time from recovery (in days) and $\operatorname{IgG}$ (ratio) in blood donors. Positive threshold $=1.1, n=129$. B IgG ratio according to donation ( $n=88,38$, and 17 , respectively). C IgG (ratio) in repeated donors $(n=17, p=0.6)$.
$30-39,40-49$, and 50-65): no significant difference was observed ( $4.0 \pm 2.2$ vs. $4.0 \pm 3.0$ vs. $3.4 \pm 2.9$ vs. $4.1 \pm 3.0$, respectively, $p=0.8$ ) (shown in Fig. 2). We then analyzed the correlation between IgG results related to time (in days) from recovery and did not show significant correlation (shown in Fig. 3A). As the IgG level may vary from donation to donation, we analyzed IgG between donation 1 and 2, and between donation 2 and 3, for donors who returned to donation once or twice, respectively. Average IgG ratio did not significantly vary between donations ( $3.9 \pm 2.7$ vs. $4.01 \pm 2.2$ vs. $39 \pm 2.0, p=0.8)$. Results are presented in Figure 3B. When we focused on repeated blood donors, no significant differences were observed either (shown in Fig. 3C).

No adverse reaction was reported during plasma apheresis or after donation by donors.

\section{Discussion}

We described the recruitment and characteristics of CP donors from (French Military) Health Service workers who experienced mild to moderate COVID-19 and from whom several donations have been collected.

We are the first to show that repeated donation is an effective way to collect more $\mathrm{CP}$ with a neutralizing antibody titer of 1:40. Nine out of $13(69 \%)$ had a neutralizing antibody titer of 1:40 after a median time of 54 (49-61) days after recovery. In an English study, the median neutralizing antibody titer significantly decreased from 1:70 in those donating within 40 days from diagnosis to $1: 43$ and 1:22 in those donating within at least 50 days [18]. We showed that donors could be sampled at least 3 times without complication in the first 70 days after COVID-19 recovery. 
We chose a threshold of 1:40 for neutralizing antibody level. Currently, no threshold has been proven to be effective in a prospective study. A minimum neutralizing titer of 1:100 and 1:160 was provided for clinical use in two other studies $[18,19]$. Based on prior SARS-CoV-1 studies, a titer of $\geq 1: 40$, assessed by cytopathic effect based on virus neutralizing tests, was supposed to be relevant [20, 21]. The choice was made to select a low threshold in order not to be too restrictive in donor recruitment.

We did not require clinical criteria or proof of biological recovery prior to donor retrieval. A kinetic study of viral shedding and antibody detection reported the presence of higher IgG and IgM antibody titers in severe patients [22], and in some clinical trials [19, 23], only patients with severe diseases were recruited in order to target high levels of neutralizing antibody titers. In an English study describing 436 donors, the highest levels of neutralizing antibodies were found in donors hospitalized, in older patients, and those who donated $<60$ days from diagnosis [18]. In our work, we show that $76 \%$ of donors had neutralizing antibody levels in their first donation although none of them had a severe form and only 2 were treated as inpatients. This is consistent with the English study in which only $10 \%$ had been hospitalized [18]. We show that donors who do not have severe forms are suitable potential donors and clinical severity may limit the donation of CP. Indeed, individuals with mild and severe disease produced neutralizing IgG to SARS$\mathrm{CoV}-2$ up to 10 days after disease onset, and most patients with severe form are again in the intensive care unit [24].

All donors included recovered for more than 14 days and therefore were no longer considered contagious. There is evidence for a significant decline in neutralizing antibody levels over time $[18,25,26]$. Some authors included donors only if they had 2 subsequent negative COVID-19 nasopharyngeal PCR $[19,23]$. We emphasize that waiting for PCR negativation can be a waste of time because neutralizing antibody levels are highest just after clinical recovery. Furthermore, there is evidence of persistent viral shedding in nasopharyngeal secretions more than 2 weeks after resolution of symptoms from confirmed COVID-19 infection [27]. The 14-day delay after cure seems to be a suitable time for the first donation.

If found to be inadequate, we proposed that donors donate their plasma. This modality of recruitment and patient interest in helping the society can be an opportunity to collect plasma for trauma patients, for example.

In conclusion, we showed that the recruitment of young donors with a mild to moderate COVID-19 course is an efficient possibility to collect CP with a satisfactory level of neutralizing antibodies. Repeated donations is a well-tolerated and effective way of CP collection.

\section{Acknowledgements}

We would like to warmly thank blood donors for their generous participation. We also would like to thank healthcare workers involved in blood collection and in blood products processing who did an incredible work in a short and troubling time.

\section{Statement of Ethics}

All subjects have given their written informed consent, and the study protocol was approved by the institute's committee on human research.

\section{Conflict of Interest Statement}

The authors declare no conflicts of interest.

\section{Funding Sources}

None.

\section{Author Contributions}

1. P.L. Conan, C. Ficko and C. Martinaud participated in research design and data analysis and wrote the manuscript.

2. All authors contributed to the patients' care and reviewed and approved the final manuscript.

\section{References}

1 Zhou F, Yu T, Du R, Fan G, Liu Y, Liu Z, et al. Clinical Course and Risk Factors for Mortality of Adult Inpatients With COVID-19 in Wuhan, China: A Retrospective Cohort Study. Lancet (London, England). 2020; 395(10229).

2 Chen T, Wu D, Chen H, Yan W, Yang D, Chen G, et al. Clinical characteristics of 113 deceased patients with coronavirus disease 2019: retrospective study. BMJ. 2020;368: m1091.
3 Siordia JA, Bernaba M, Yoshino K, Ulhaque A, Kumar S, Bernaba M, et al. Systematic and Statistical Review of Coronavirus Disease 19 Treatment Trials. SN Compr Clin Med. 2020 Aug;2(8):1120-31.

4 The Recovery Collaborative Group. Dexamethasone in Hospitalized Patients with Covid-19 - Preliminary Report. N Engl J Med. 2020.

5 Arabi YM, Hajeer AH, Luke T, Raviprakash $\mathrm{K}$, Balkhy H, Johani S, et al. Feasibility of using convalescent plasma immunotherapy for MERS-CoV infection, Saudi Arabia. Emerg Infect Dis. 2016.
6 Mair-Jenkins J, Saavedra-Campos M, Baillie JK, Cleary P, Khaw FM, Lim WS, et al. The effectiveness of convalescent plasma and hyperimmune immunoglobulin for the treatment of severe acute respiratory infections of viral etiology: A systematic review and exploratory meta-analysis. J Infect Dis. 2015 Jan 1;211(1):80-90.

$7 \mathrm{Wu}$ XX, Gao HN, Wu HB, Peng XM, Ou HL, Li LJ. Successful treatment of avian-origin influenza A (H7N9) infection using convalescent plasma. Int J Infect Dis. 2015 Dec;41:3-5. 
8 Garraud O. Use of convalescent plasma in Ebola virus infection. Transfus Apher Sci. 2017 Feb;56(1):31-34.

9 Keller MA, Stiehm ER. Passive immunity in prevention and treatment of infectious diseases. Clin Microbiol Rev. 2000 Oct;13(4): 602-14.

10 Graham BS, Ambrosino DM. History of passive antibody administration for prevention and treatment of infectious diseases. Curr Opin HIV AIDS. 2015 May;10(3):129-34.

11 Piyush R, Rajarshi K, Khan R, Ray S. Convalescent plasma therapy: a promising coronavirus disease 2019 treatment strategy. Open Biol. 2020 Sep;10(9):200174.

12 Hueso T, Pouderoux C, Péré H, Beaumont A-L, Raillon L-A, Ader F, et al. Convalescent plasma therapy for B-cell depleted patients with protracted COVID-19 disease. Blood. 2020 Nov; 136(20):2290-2295.

13 Bakhtawar N, Usman M, Khan MMU. Convalescent Plasma Therapy and Its Effects On COVID-19 Patient Outcomes: A Systematic Review of Current Literature. Cureus. 2020 Aug;12(8):e9535.

14 Shah VK, Firmal P, Alam A, Ganguly D, Chattopadhyay S. Overview of Immune Response During SARS-CoV-2 Infection: Lessons From the Past. Front Immunol. 2020 Aug;11: 1949.
15 Gniadek TJ, Donnersberger D. COVID-19 convalescent plasma donor recruitment: beware the Faustian bargains. Transfusion. 2020 Jul;60(7):1643-4.

16 Roehrig JT, Hombach J, Barrett ADT. Guidelines for plaque-reduction neutralization testing of human antibodies to dengue viruses. Viral Immunol. 2008 Jun;21(2):123-32.

17 Gallian P, Pastorino B, Morel P, Chiaroni J, Ninove L, de Lamballerie X. Lower prevalence of antibodies neutralizing SARS-CoV-2 in group O French blood donors. Antiviral Res. 2020 Sep;181:104880.

18 Harvala H, Mehew J, Robb ML, Ijaz S, Dicks $\mathrm{S}$, Patel $\mathrm{M}$, et al. Convalescent plasma treatment for SARS-CoV-2 infection: analysis of the first 436 donors in England, 22 April to 12 May 2020. Euro Surveill. 2020;25(28):1-7.

19 Li L, Tong X, Chen H, He R, Lv Q, Yang R, et al. Characteristics and serological patterns of COVID-19 convalescent plasma donors: optimal donors and timing of donation. Transfusion. 2020 Aug;60(8):1765-72.

20 Zhang JS, Chen JT, Liu YX, Zhang ZS, Gao H, Liu Y, et al. A serological survey on neutralizing antibody titer of SARS convalescent sera. J Med Virol. 2005 Oct;77(2):147-50.

21 Tiberghien P, de Lamballerie X, Morel P, Gallian P, Lacombe K, Yazdanpanah Y. Collecting and evaluating convalescent plasma for
COVID-19 treatment: why and how? Vox Sang. 2020 Aug;115(6):488-494.

22 Wang Y, Zhang L, Sang L, Ye F, Ruan S, Zhong B, et al. Kinetics of viral load and antibody response in relation to COVID-19 severity. J Clin Invest. 2020 Oct;130(10):52355244.

23 Li L, Yang R, Wang J, Lv Q, Ren M, Zhao L, et al. Feasibility of a pilot program for COVID-19 convalescent plasma collection in Wuhan, China. Transfusion. 2020 Aug;60(8):1773-7.

24 Casadevall A, Joyner MJ, Pirofski L-A. SARSCoV-2 viral load and antibody responses: the case for convalescent plasma therapy. J Clin Invest. 2020 Oct;130(10):5112-5114.

25 Brochot E, Demey B, Touze A, Belouzard S, Dubuisson J, Schmit J-L, et al. Anti-Spike anti-Nucleocapsid and neutralizing antibodies in SARS-CoV-2 hospitalized patients and asymptomatic carriers. Medrxiv. 2020.

26 Wang X, Guo X, Xin Q, Pan Y, Hu Y, Li J, et al. Neutralizing Antibodies Responses to SARS-CoV-2 in COVID-19 Inpatients and Convalescent Patients. Clin Infect Dis. 2020 Dec;71(10):2688-2694.

27 Hartman WR, Hess AS, Connor J. Persistent viral RNA shedding after COVID-19 symptom resolution in older convalescent plasma donors. Transfusion. 2020 Oct;60(10):21892191 